\title{
Oral Valproate Sodium as an alternative to Benzodiazepine in the treatment of Catatonia - A Case report
}

\author{
Jacob C. Maier ${ }^{a}$ Daniel Rapport $^{b}$ Alex McCormick ${ }^{c}$ and Chandani Lewis ${ }^{b}$
}

Coresponding author(s): Chandani.lewis@utoledo.edu

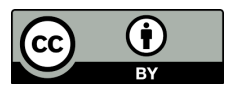

${ }^{a}$ The University of Toledo School of Medicine 3000 Arlington Ave. Toledo, OH 43614, USA, ${ }^{b}$ The University of Toledo Medical Center Department of Psychiatry 3000 Arlington Ave. Toledo, $\mathrm{OH} 43614$, USA, and ${ }^{c}$ Northcoast Behavioral Healthcare 1756 Sagamore Rd Northfield, $\mathrm{OH} 44067, \mathrm{USA}$

\begin{abstract}
Alternative therapies are necessary to treat catatonia in patients with comorbidities that are not amenable to therapy with benzodiazepines or ECT. This is a patient with schizophrenia with catatonic features and a history of polysubstance abuse. Consequently, he was not a candidate for treatment with benzodiazepines, so an alternative needed to be found. GABAergic medications have been used previously as alternatives to benzodiazepines and ECT. In this case we chose sodium valproate, due to its cross-reaction with GABAergic systems. There are five reported cases using sodium valproate. Three of which were treated with intravenous valproate, while the remaining two do not specify the route of administration. We present a case where oral sodium valproate was used successfully for both acute and long-term catatonic treatment. To our knowledge, no other report has looked at both acute and long-term treatment with sodium valproate. Oral sodium valproate can be considered for patients with substance use disorders COPD, sleep apnea or myasthenia gravis in which benzodiazepines are contraindicated and where ECT is not an option for treatment.
\end{abstract}

Oral Valproate Sodium I alternative I Benzodiazepine I treatment of Catatonia

C atatonia is a complex neuropsychiatric disorder that complicates numerous medical and psychiatric illnesses. The disorder requires urgent medical attention as it can be life-threatening if left untreated. Benzodiazepines and electroconvulsive therapy (ECT) are considered the first line therapies for catatonia. However, in situations where benzodiazepines are contraindicated and ECT is not available, catatonia must be managed with a second-line therapy. Valproic acid, sodium valproate or divalproex sodium (sodium valproate) as a treatment for catatonia is not well documented. A literature search yielded only 5 cases of this disorder being successfully treated mainly with parenteral sodium valproate (1-4). We present the case of a 30-year-old schizophrenic patient with catatonic features that was successfully treated with oral sodium valproate. The results suggested that oral sodium valproate is an acceptable and important alternative therapy for catatonia when ECT is not available and benzodiazepines are contraindicated. Benzodiazepines were acceptable for acute management of this patient. However, to maintain his abstinence and promote his sobriety, he needed to be placed in a substance abuse rehabilitation facility. For this reason, benzodiazepines could not be prescribed to this patient. In this patient and others, for whom benzodiazepines are contraindicated due to comorbid conditions such as chronic obstructive pulmonary disease (COPD), sleep apnea and myasthenia gravis, oral sodium valproate may be helpful.

\section{Case Report}

Patient Information

Age: 30 years old. Gender: Male. Ethnicity: African American. Related Medical Problems: Schizophrenia with catatonic features, polysubstance abuse disorder.

Submitted: 24/11/2020, published: 24/02/2021.

Freely available online through the UTJMS open access option 


\section{Objective for Case Reporting}

To discuss oral sodium valproate as an alternative to benzodiazepine or ECT therapy in the treatment of catatonia in schizophrenia and to explore scenarios in which benzodiazepines and ECT therapy are contraindicated.

\section{Case}

The patient was a 30-year-old African American male, with a history of schizophrenia with catatonic features. He was admitted to the state hospital for violating his conditional release after relapsing on alcohol, marijuana, and crack cocaine.

The patient had been previously treated for catatonia with oral lorazepam, but this had to be discontinued in order for him to be admitted to a rehabilitation center for chemical dependence. Previously, his catatonic symptoms consisted of stupor, negativism, mutism, and rigidity. During his intake at the state hospital, the patient had multiple staring episodes lasting 5-10 minutes throughout his interviews and was often slow to respond to questions. His medications included haldol decanoate $200 \mathrm{mg}$ Q4/week, oral quetiapine $200 \mathrm{mg}$ daily, oral propranolol $20 \mathrm{mg}$ twice daily, oral benztropine $1 \mathrm{mg}$ twice daily for the management of antipsychotic induced extra pyramidal side effects, especially akathisia. There was no evidence of acute dystonic reactions, signs of Parkinson's, neuroleptic malignant syndrome or seizures and his laboratories were within normal limits. Since ECT was not available at this state hospital and he could not be restarted on lorazepam, we sought an alternative therapy. A literature search yielded a case of catatonia that was successfully treated with sodium valproate, so we decided to treat him with oral sodium valproate.

MS was started on a $1000 \mathrm{mg}$ dose of sodium valproate $(500 \mathrm{mg}$ twice daily) which provided partial improvement. Initially, his staring episodes ceased, but after a period of 8 days he reported an episode where his eyes focused on the door handle for about 3 hours. It is unlikely that these episodes were seizures as there was no history of seizures in this patient and the patient did not exhibit a post ictal state. EEG was not done. This prompted us to increase the oral dose to $1500 \mathrm{mg}$ daily, which resulted in resolution of his symptoms within 3 days. His blood levels of sodium valproate ranged from 74$79 \mathrm{mcg} / \mathrm{ml}$ and his catatonic symptoms were completely resolved at discharge.

\section{Discussion}

Catatonia is a movement disorder typically related to schizophrenia or other psychiatric symptoms. In order to make the diagnosis, patients must display 3 of 12 symptoms listed in the DSM-5. These features are stupor, mutism, waxy flexibility, negativism, posturing, mannerisms, stereotypy, agitation, echolalia and echopraxia (5). Our patient met DSM-5 criteria for catatonia by demonstrating stupor, mutism, posturing, negativism, and agitation.

Treating catatonia is essential. Complications associated with stuporous catatonia include dehydration, starvation, urinary tract infections and pneumonia which can have poor prognoses if not addressed. Similarly, there are severe complications associated with excited catatonia which include hyperthermia and rhabdomyolysis. Higher doses of neuroleptics are neither safe nor effective and may lead to worse complications like neuroleptic malignant syndrome (NMS). NMS is the most severe sequelae of catatonia and can be deadly (6). While rates of NMS have declined with the development of modern antipsychotic therapies, lethal catatonia remains a potentially grave complication if catatonic features are not appro- priately managed $(7,8)$.

Benzodiazepines have long been the first-line therapy for treating catatonia, demonstrating effectiveness in $70 \%$ of cases regardless of cause. $(9,10)$. Rasmussen et al., postulate that catatonia's symptomatic overlap with parkinsonism and it's responsiveness to benzodiazepines indicate an underlying pathology of the GABAergic system in the basal ganglia (11).

While therapy with benzodiazepines is considered first-line, electroconvulsive therapy (ECT) is also a widely accepted treatment for catatonia $(10,12)$. However, ECT is not always available and requires clear consent to be given by the patient (11). Most catatonic patients do not have capacity to consent and therefore are unable to discuss ECT or to its administration.

Anti- convulsants due to their effect on GABA-nergic system have shown effectiveness in treating catatonic symptoms (10). There are case reports of anti-convulsants like sodium valproate, levetiracetam, topiramate, and carbamazepine used to treat catatonia. Parenteral form of sodium valproate was most commonly used anticonvulsant.

The existing literature proposes many hypotheses on how sodium valproate exerts its effect on the GABA system. Kerwin et al. suggest that the drug increases the concentration of GABA by inhibiting GABA-aminotransferase, while Tunnicliff contends that sodium valproate also increases GABA synthesis and potentiates post-synaptic GABAergic effects $(13,14)$. While the exact mechanism of action of sodium valproate is unclear, it is known that the drug enhances central nervous system GABA levels and neuronal GABA responsiveness, working on a similar pathway as benzodiazepines $(14,15)$. Considering that sodium valproate has been used in other cases for benzodiazepine detoxification, the concern of using it in a patient with substance abuse is mitigated. The advantage of using sodium valproate is that it is not a controlled substance. In this case, our patient could not be admitted to a chemical dependency facility on any type of controlled substance, making sodium valproate an ideal substitute.

While researching alternative therapies, a literature search yielded 4 case-reports detailing the treatment of catatonia with sodium valproate. An additional report described a 46-year-old woman with a history of alcohol use disorder that had "affective" catatonic symptoms refractory to typical antipsychotic treatment (2). The patient was started on a regimen of $1000 \mathrm{mg}$ of sodium valproate daily which alleviated her catatonic symptoms. A case presented by Bowers et al. described a patient with treatment refractory schizophrenia, catatonic subtype, whose healthcare power of attorney refused to consent to ECT (1). That patient was started on a $4000 \mathrm{mg}$ per day intravenous dose of sodium valproate which was tapered down to $1800 \mathrm{mg}$ per day by the fourth day. That patient was discharged on $900 \mathrm{mg}$ of oral sodium valproate daily and his catatonic features resolved over the subsequent 10 months.

Our case is significant because he was treated both acutely and long term with oral sodium valproate. There are no other reports of oral sodium valproate that address both acute episodes and longterm prevention to our knowledge. Our findings indicate that oral sodium valproate could be used in the management of catatonia in patient with schizophrenia. The advantage of using sodium valproate is that it is available in oral and in parenteral form and it is not a controlled substance.

This patient was treated with a conventional dose of oral sodium valproate at therapeutic blood levels, which was well tolerated without side effects. Sodium valproate is not without its risks and limita- 
tions. While uncommon, hypothyroidism, thrombocytopenia, liver failure, pancreatitis and folic acid depletion are among the potential adverse effects. One report notes that sodium valproate can induce hyper-ammonemic encephalopathy which can present like catatonic features (16).

\section{Conclusion}

In situations where benzodiazepines and ECT are either unavailable, contraindicated, or ineffective, we suggest that oral sodium valproate may be an adequate alternative. Future studies should focus on the mechanism of valproic acid as well as long

1. Kruger $S$ \& Braunig $P$ (2001) Intravenous valproic acid in the treatment of severe catatonia. J Neuropsychiatry Clin Neurosci 13(2):303-304.

2. Bowers $R \&$ Ajit SS (2007) Is there a role for valproic acid in the treatment of catatonia? J Neuropsychiatry Clin Neurosci 19(2):197-198.

3. Yoshida I, Monji A, Hashioka S, Ito M, \& Kanba S (2005) Prophylactic effect of valproate in the treatment for siblings with catatonia: a case report. $J$ Clin Psychopharmacol 25(5):504-505.

4. DelBello MP, Foster KD, \& Strakowski SM (2000) Case report: treatment of catatonia in an adolescent male. $J$ Adolesc Health 27(1):69-71.

5. Tandon R, et al. (2013) Catatonia in DSM-5. Schizophr Res 150(1):26-30.

6. White DA \& Robins AH (2000) An analysis of 17 catatonic patients diagnosed with neuroleptic malignant syndrome. CNS Spectr 5(7):58-65.

7. Mann SC, et al. (1986) Lethal catatonia. Am J Psychiatry 143(11):1374-1381.

8. Mann SC, Caroff SN, Bleier HR, Antelo RE, \& Un H (1990) Electroconvulsive Therapy of the Lethal Catatonia Syndrome. Convuls Ther 6(3):239-247.

9. Hawkins JM, Archer KJ, Strakowski SM, \& Keck PE (1995) Somatic treatment of catatonia. Int J Psychiatry Med 25(4):345-369. term outcomes and side effects in catatonic patients.

\section{Conflict of interest}

Authors declare no conflict of interest.

\section{Authors' contributions}

JCM and CL wrote the manuscript, DR and CL revised the manuscript, AM provided patient information. All authors have read and approved the final document.

10. Sienaert P, Dhossche DM, Vancampfort D, De Hert M, \& Gazdag G (2014) A clinical review of the treatment of catatonia. Front Psychiatry 5:181.

11. Rasmussen SA, Mazurek MF, \& Rosebush PI (2016) Catatonia: Our current understanding of its diagnosis, treatment and pathophysiology. World J Psychiatry 6(4):391-398.

12. Luchini $F$, et al. (2015) Electroconvulsive therapy in catatonic patients: Efficacy and predictors of response. World J Psychiatry 5(2):182-192.

13. Tunnicliff $G$ (1999) Actions of sodium valproate on the central nervous system. $J$ Physiol Pharmacol 50(3):347-365.

14. Kerwin RW \& Taberner PV (1981) The mechanism of action of sodium valproate. Gen Pharmacol 12(2):71-75.

15. Baldino F, Jr. \& Geller HM (1981) Sodium valproate enhancement of gammaaminobutyric acid (GABA) inhibition: electrophysiological evidence for anticonvulsant activity. J Pharmacol Exp Ther 217(2):445-450.

16. Perez-Esparza R, Onate-Cadena N, Ramirez-Bermudez J, \& Espinola-Nadurille M (2018) Valproate-induced Hyperammonemic Encephalopathy Presenting as Catatonia. Neurologist 23(2):51-52. 